\title{
Effects of Fatigue on Immune Function in Nurses Performing Shift Work
}

\author{
Makie Nagai ${ }^{1}$, Yuko Morikawa ${ }^{1}$, Kazuyo Kitaoka ${ }^{2}$, Koshi NaKamura ${ }^{1}$, Masaru Sakurai ${ }^{1}$, \\ Muneko NishiJo ${ }^{1}$, Yuko Hamazaki ${ }^{2}$, Shoko Maruzeni ${ }^{1}$ and Hideaki NaKagawa ${ }^{1}$ \\ ${ }^{1}$ Department of Epidemiology and Public Health and ${ }^{2}$ School of Nursing, Kanazawa Medical University, Japan
}

\begin{abstract}
Effects of Fatigue on Immune Function in Nurses Performing Shift Work: Makie NAGAl, et al. Department of Epidemiology and Public Health, Kanazawa Medical University-Objectives: We investigated the effects of fatigue on NK cell function and lymphocyte subpopulations in nurses performing shift work using a longitudinal design. Methods: Fiftyseven female nurses engaged in shift work at a hospital in Japan were selected for our study cohort. The hospital used a counterclockwise rotating three-shift system. Night shifts followed day shifts after a sevenhour interval. Immune parameters measured at the beginning of the day shift through to the end of the night shift were compared between two groups stratified by their level of fatigue. Statistical differences were evaluated after adjusting for baseline immune values and other demographic features. Results: Subjective feelings of fatigue increased progressively from the beginning of day shifts to the end of night shifts. From the beginning of day shifts to the end of night shifts, NK cell activity and $\mathrm{CD} 16^{+} \mathrm{CD} 56^{+}$Iymphocytes decreased, while $\mathrm{CD}^{+}$and $\mathrm{CD}^{+}$lymphocytes increased. The group with the greater increase in fatigue showed a larger decrease in NK cell activity and a larger increase in $\mathrm{CD}^{+}$lymphocytes when compared with the group reporting less fatigue. These findings did not change after adjusting for demographic factors and sleep hours. Conclusion: Our data suggest that shift work has deleterious effects on NK cell function and that the effects depend on the degree of fatigue. Proper management of shift work may lessen fatigue in workers and also ameliorate many health problems experienced by shift workers.

(J Occup Health 2011; 53: 312-319)
\end{abstract}

Received Dec 25, 2010; Accepted May 26, 2011

Published online in J-STAGE Jul 20, 2011

Correspondence to: M. Nagai, School of Nursing, Kanazawa Medical University, 1-1 Daigaku, Uchinada, Kahoku-gun, Ishikawa 920 0293, Japan (e-mail:m-long@kanazawa-med.ac.jp)
Key words: Fatigue, Immune function, Lymphocyte subsets, NK cell activity, Shift work

The relationship between shift work and cancer has been a recent subject of interest. The International Agency for Research on Cancer (IARC) concluded that shift work that involves circadian disruption is a probable carcinogenic risk factor for humans (group 2A) for hormone-dependent cancers, such as breast cancer ${ }^{1)}$. Among the IARC findings is that cancer risk increases due to the disturbance of biological circadian rhythms, which results in decreased secretion of melatonin and changes in hormonal secretion patterns and immunological function ${ }^{2}$. A reduction in melatonin production leads to immune suppression, including a reduction in the number of natural killer (NK) cells and cytotoxic lymphocytes and a decrease in proinflammatory cytokines ${ }^{3}$.

There have been quite a few studies examining the effects of shift work on immunological function. Nakano et al. ${ }^{4)}$ reported that lymphocyte proliferation in shift workers was decreased compared with that of fixed-hour daytime workers in a wholesale market and manufacturing company. Okamoto et al. ${ }^{5)}$ also reported that natural killer (NK) cell activity in rotating shift workers was lower than in workers with fixed daytime schedules in a study of medical doctors. However, these studies utilized cross-sectional designs and a direct, causal relationship between shift work and immune function has not been demonstrated.

There are several laboratory studies that have used simulated night work to investigate the effects of partial or total sleep deprivation on immune function ${ }^{6-11)}$. Both enhancing and suppressive effects on NK cell activity and lymphocyte subsets have been reported. It has been suggested that human subjects exposed to laboratory stressors exhibit transient increases in immune function, but that more intense and/or long-lived stressors impair the same measures of immune function ${ }^{12}$. Furthermore, both natural and specific immunity are impacted by chronic and acute stressors ${ }^{13)}$. We hypothesized that 
Table 1. Demographic characteristics of the nurses working in a Japanese psychiatric hospital

\begin{tabular}{ll}
\hline & $\mathrm{n}=57$ \\
\hline Age (yr) & $42.1(8.4)$ \\
Years working as a nurse & $20.5(8.7)$ \\
Years of shift work & $19.0(8.4)$ \\
Position, manager/subordinate & $15.8 / 84.2$ \\
Wards for patients with schizophrenia and schizophreniform disorder/dementia & $50.9 / 49.1$ \\
Marital status, single/married & $12.3 / 87.7$ \\
Cigarette smoking habits, current/former or never & $5.3 / 94.7$ \\
\hline
\end{tabular}

Values are expressed as means (standard deviation) or percentages of participants in the category.

greater levels of sleep deprivation or circadian rhythm disruption will have a greater impact on immunity/ numerous immune variables.

Based on this hypothesis, we used a longitudinal study design to investigate the effects of shift work-related fatigue level on immune function among nurses engaged in shift work. The impact of shift work as a stressor was measured by subjective feelings of fatigue. The relationship between increased levels of fatigue and changes in immune function during a 24-hour observational period including a night shift was examined. Immune function was evaluated by assessing NK cell activity and various $\mathrm{T}$ lymphocyte subpopulations that have been demonstrated to be important for anticancer immunity.

\section{Methods}

\section{Subjects}

This study was conducted in a public hospital for psychiatric disorders (400 beds, 150 nurses) in Ishikawa, Japan. One hundred and five nurses (28 males and 77 females) aged 22-59 participated in this study. Among them, 61 female nurses engaged in shift work were our study subjects. Four subjects who took medications known to affect the immune system (medications for allergy or rheumatism) were excluded, leaving 57 subjects remaining. All subjects were physically healthy and not suffering from overt infections. Table 1 presents demographic features of our study participants. The mean age was $42.1 \mathrm{yr}$ (range of 23-59 yr, standard deviation (SD) of $8.4 \mathrm{yr}$ ), and the mean number of years of shift work was 19.0 (range of $0.5-39 \mathrm{yr}, \mathrm{SD}$ of $8.4 \mathrm{yr}$ ). The percentages of participants engaged in a managerial position, married and a current smoker were 15.8, 87.7 and $5.3 \%$, respectively. This study was approved by the Ethics Committee for Epidemiologic Research at Kanazawa Medical University (Ishikawa Prefecture, Japan). All participants provided informed consent in order to participate in our study.

\section{Survey}

The target hospital used a counterclockwise rotating three-shift system. The ordinal order of each shift was day (08:30-17:15), night (00:30-09:15), evening (16:30$01: 15)$ and off. The interval between day shifts and night shifts was approximately seven hours. Taking a nap during night shifts was not officially permitted.

Blood samples were collected from subjects on two consecutive mornings. Sampling was carried out at the beginning of the daytime shift on the first day (8:00-9:00), and the second day's sample was collected at the end of the night shift (9:00-10:00). The participants were not allowed to smoke, eat or move vigorously within 30 minutes of blood sampling. Twelve-hour presampling sleep diaries and questionnaires exploring each subject's fatigue immediately before and immediately after each shift were completed by each study participant.

NK cell activity and lymphocyte surface antigens were assayed by BML Inc. (Tokyo, Japan). NK cell activity was measured by a standard chromium release assay ${ }^{14}$. The effector: target (E:T) ratios were $10: 1$ and $20: 1$. Percent cytotoxicity was calculated for each E:T ratio. NK cells were measured by two-color flow cytometry using anti-CD16 and anti-CD56. Single-color flowcytometry was used to assay for lymphocytes expressing CD3, CD4, and CD8 to measure mature T cells, helper T cells, and cytotoxic $\mathrm{T}$ cells, respectively.

Fatigue was evaluated at four time points (the beginning of day shift, the end of day shift, the beginning of night shift and the end of night shift) by a questionnaire for work-related fatigue feelings, the "Jikaku-sho shirabe" designed by the Industrial Fatigue Research Committee of the Japan Society of Occupational Health ${ }^{15,16)}$. This questionnaire was developed to evaluate trends in feelings of fatigue due to work, and factorial validity and internal consistency have been verified ${ }^{17}$. The questionnaire consists of 25 subjective fatigue symptom items that are categorized into five factors with five items in each: "drowsiness" indicating sleepiness, "instability" indicating mental fatigue, "uneasiness" indicating symptoms like autonomic imbalance, "local pain or dullness" measuring physical complaints, and "eyestrain" indicating asthenopia. For each item, respondents were requested to estimate the 
Table 2. Changes in subjective feelings of fatigue due to shift work, from the beginning of day shifts to the end of night shifts

\begin{tabular}{|c|c|c|c|c|c|c|c|c|c|c|c|}
\hline Fatigue & $\begin{array}{r}\text { Beg } \\
\text { da }\end{array}$ & $\begin{array}{l}\text { nning of } \\
\text { / shift } t^{\text {a) }}\end{array}$ & & $\begin{array}{l}\text { nd of } \\
\text { shift }^{\text {b) }}\end{array}$ & $\begin{array}{r}\text { Beg } \\
\text { nig }\end{array}$ & $\begin{array}{l}\text { nning of } \\
\text { t shift }\end{array}$ & $\begin{array}{r}\mathrm{E} \\
\text { nig }\end{array}$ & $\begin{array}{l}\text { ad of } \\
t \text { shift }^{d)}\end{array}$ & $a / b$ & $\mathrm{~b} / \mathrm{c}$ & $\mathrm{c} / \mathrm{d}$ \\
\hline Drowsiness & 6.0 & $5.0-18.0$ & 8.0 & $5.0-25.0$ & 12.0 & $5.0-24.0$ & 13.0 & $5.0-25.0$ & $* *$ & $* *$ & $* *$ \\
\hline Instability & 6.0 & $5.0-14.0$ & 7.0 & $5.0-21.0$ & 7.0 & $5.0-17.0$ & 9.0 & $5.0-23.0$ & $* *$ & & $* *$ \\
\hline Uneasiness & 6.0 & $5.0-16.0$ & 6.0 & $5.0-17.0$ & 7.0 & $5.0-23.0$ & 8.0 & $5.0-23.0$ & $*$ & $* *$ & $* *$ \\
\hline Local pain or dullness & 6.0 & $5.0-19.0$ & 8.0 & $5.0-25.0$ & 7.0 & $5.0-20.0$ & 9.0 & $5.0-25.0$ & $* *$ & $* *$ & $* *$ \\
\hline Eyestrain & 5.0 & $5.0-19.0$ & 7.0 & $5.0-22.0$ & 8.0 & $5.0-20.0$ & 11.0 & $5.0-25.0$ & $* *$ & & $* *$ \\
\hline
\end{tabular}

Fatigue feelings are evaluated by a questionnaire for work-related fatigue feelings, the "Jikaku-sho shirabe" of the Industrial Fatigue Research Committee of the Japan Society of Occupational Health. Values are expressed as medians (range). Statistical differences were evaluated by the Wilcoxon signed-rank sum test. * and **: $p<0.05$ and $p<0.01$, respectively.

intensity of their feelings as "disagree completely," "agree slightly," "agree somewhat," "agree considerably" and "agree strongly." These five intensities were assigned values of 1 to 5 points, respectively, and summed within each factor. Therefore, the range of possible scores for each factor is 5 to 25 points, and a higher score means greater fatigue. Cronbach's alpha for the five factors was greater than 0.7 , except for "instability" $(0.67)$, indicating a high degree of internal consistency in our study.

\section{Statistical analysis}

Fatigue scores from the beginning of day shifts to the end of night shifts were tested by Wilcoxon signed-rank sum tests. The values were compared between two groups of subject characteristics, age group (23-43 vs. 44-59), hours of sleep before night shift (0-2.0 h vs. 2.5-4.0 h), marital status (married vs. single) and ward assignment (wards for patients with schizophrenia and schizophreniform disorder vs. wards for patients with dementia), by $t$-test. Comparisons of immune parameters between the beginning of day shifts and the end of night shifts were carried out by paired $t$-test. Changes in immune parameters from the beginning of day shifts to the end of night shifts were compared between two groups divided by their increased level of fatigue (small vs. large). The comparison was carried out in each of the five fatigue factors described above. Statistical differences in immune parameters between two groups were evaluated by simple comparison ( $t$-test) and by analysis of covariance using baseline immune values and other demographic features as covariates.

Statistical analyses were performed using SPSS version 18 (SPSS Inc, Chicago, IL, USA).

\section{Results}

Table 2 shows the changes in scores of subjective feelings of fatigue from the beginning of day shifts to the end of night shifts. All fatigue scores increased progressively from the beginning of day shifts to the end of night shifts. The scores at the beginning of night shifts were significantly higher than those at the end of day shifts for all factors except for "local pain or dullness" and "eyestrain," as determined by Wilcoxon signed-rank sum tests. This suggests that fatigue was not ameliorated by the interval between the day shift and night shift. Increased levels of fatigue from the beginning of day shifts to the end of night shifts were compared between two groups of demographic features such as age, marital status, ward assignment and hours of sleep during the interval (Table 3 ). The subjects who were older, married and experienced fewer sleep hours tended to show greater fatigue, although the observed differences did not reach statistical significance.

Table 4 shows changes in immune parameters from the beginning of day shifts to the end of night shifts. Since NK cell activity at the two different E:T ratios tested showed similar findings, only the results of the 20:1 E:T ratio are shown. For all groups examined, NK cell activity and $\mathrm{CD} 16^{+} \mathrm{CD} 56^{+}$lymphocytes were significantly lower at the end of night shifts than at the beginning of day shifts. Conversely, $\mathrm{CD}^{+}$and $\mathrm{CD}^{+}$lymphocyte counts were significantly higher at the end of night shifts than at the beginning of day shifts. There were significant differences in some parameters between two groups of demographic features such as age, marital status, ward assignment and sleep hours (Table 5). The decrease in NK cell activity was larger in married subjects and in subjects assigned to wards for patients with dementia. The increases in $\mathrm{CD}^{+}$, $\mathrm{CD}^{+}$and $\mathrm{CD}^{+}$lymphocytes were larger in younger subjects than in older subjects.

Changes in immune parameters from the beginning of day shifts to the end of night shifts were compared between groups differentiated by the magnitude of the increase in fatigue (small vs. large). Table 6 shows the results for three of the five fatigue factors, drowsiness, instability, and local pain or dullness, which had significant differences in some immune parameters between the fatigue groups. Statistically significant differences in immune parameters between the different dichotomous fatigue groups were evaluated by simple comparison ( $t$-test) and by analysis 
Table 3. Changes in five fatigue factors, from the beginning of day shifts to the end of night shifts, grouped by age, marital status, ward assignment and hours of sleep before the night shift

\begin{tabular}{|c|c|c|c|c|c|c|}
\hline & \multirow{2}{*}{$\begin{array}{l}\text { Number of } \\
\text { subjects }\end{array}$} & \multicolumn{5}{|c|}{ Increase of feelings of fatigue } \\
\hline & & Drowsiness & Instability & Uneasiness & $\begin{array}{l}\text { Local pain or } \\
\text { dullness }\end{array}$ & Eyestrain \\
\hline All & 57 & $7.1(5.7)$ & $3.6(4.6)$ & $3.5(4.5)$ & $3.4(4.1)$ & $5.6(4.9)$ \\
\hline \multicolumn{7}{|l|}{ Age (yr) } \\
\hline $23-43$ & 29 & $7.2(6.2)$ & $3.4(4.6)$ & $2.8(4.2)$ & $3.6(3.5)$ & $5.3(4.6)$ \\
\hline $44-59$ & 28 & $6.9(5.3)$ & $3.9(4.8)$ & $4.4(4.8)$ & $3.3(4.7)$ & $5.9(5.2)$ \\
\hline \multicolumn{7}{|l|}{ Marital status } \\
\hline Single & 10 & $4.4(5.1)$ & $1.7(2.9)$ & $1.4(3.7)$ & $2.7(2.1)$ & $3.8(3.6)$ \\
\hline Married & 47 & $7.6(5.7)$ & $4.0(4.9)$ & $4.0(4.6)$ & $3.6(4.4)$ & $6.0(5.0)$ \\
\hline \multicolumn{7}{|l|}{ Wards for patients with: } \\
\hline Dementia & 28 & $6.7(6.7)$ & $3.9(5.5)$ & $3.0(5.1)$ & $2.9(3.6)$ & $5.3(5.5)$ \\
\hline $\begin{array}{l}\text { Schizophrenia and } \\
\text { schizophreniform disorder }\end{array}$ & 29 & $7.4(4.7)$ & $3.3(3.6)$ & $4.1(3.8)$ & $3.9(4.5)$ & $5.9(4.2)$ \\
\hline \multicolumn{7}{|c|}{ Hours of sleep before the night shift } \\
\hline $0-2.0 \mathrm{~h}$ & 34 & $7.7(5.4)$ & $4.0(5.0)$ & $4.2(3.9)$ & $3.8(4.0)$ & $6.1(5.2)$ \\
\hline $2.5-4.0 \mathrm{~h}$ & 23 & $6.1(6.2)$ & $3.0(4.1)$ & $2.6(5.2)$ & $2.9(4.4)$ & $4.8(4.3)$ \\
\hline
\end{tabular}

Values are expressed as means (standard deviation) in the category. Statistical differences were evaluated by $t$-test.

Table 4. Comparison of immunological parameters between the beginning of day shifts and the end of night shifts

\begin{tabular}{lrrrr}
\hline & \multicolumn{1}{c}{ Baseline } & Endpoint & $\mathrm{t}$ value & \\
\hline NK cell activity $(\mathrm{E}: \mathrm{T}=20: 1, \%)$ & $17.0(10.1)$ & $13.7(9.7)$ & 3.9 & $* *$ \\
$\mathrm{CD}^{+}$lymphocytes (number $\left./ \mu l\right)$ & $1,208.4(397.8)$ & $1,413.7(365.8)$ & 6.6 & $* *$ \\
$\mathrm{CD} 4^{+}$lymphocytes (number $\left./ \mu l\right)$ & $736.7(291.3)$ & $860.7(282.1)$ & 6.4 & $* *$ \\
$\mathrm{CD} 8^{+}$lymphocytes $($number $/ \mu l)$ & $542.3(196.8)$ & $565.2(172.3)$ & 1.2 & \\
$\mathrm{CD} 16^{+} \mathrm{CD} 56^{+}$lymphocytes $($number $/ \mu l)$ & $292.0(196.4)$ & $222.3(123.1)$ & 3.6 & $* *$ \\
\hline
\end{tabular}

Values are expressed as means (standard deviation). Statistical differences were evaluated by paired $t$-test. * and $* *: p<0.05$ and $p<0.01$, respectively.

of covariance using baseline immune values and other demographic features. Model 1 shows the simple comparison. Model 2 shows the results after adjustment for each baseline (at the beginning of day shifts) immune variable. Model 3 shows the results after adjustment for baseline parameters, age, marital status, ward assignment and hours of sleep before the night shift. We found statistically significant decreases in NK cell activity in the group experiencing a large increase in "drowsiness" and "local pain or dullness." Although CD $16^{+}$CD56 $6^{+}$ lymphocytes tended to decrease in the group that experienced a large increase in fatigue, it did not reach statistical significance. Additionally, we found significant increases in $\mathrm{CD}^{+}$lymphocytes in the group that experienced a large increase in "drowsiness," "instability" and "local pain or dullness."

\section{Discussion}

We investigated the effect of fatigue due to shift work on immune parameters such as NK cell function and lymphocyte subsets. Our subjects were engaged in a shift work system that is typical for hospital nurses in Japan. Night shifts followed day shifts after only a seven-hour interval. Since most subjects had to deal with housework during that interval, they actually slept $0-4 \mathrm{~h}$ prior to starting the night shift. Perceived fatigue was not alleviated during that interval and accumulated until the end of the night shift. Similar results have been reported in Japan previously ${ }^{18,19)}$.

NK cell function decreased from the beginning of day shifts to the end of night shifts. These changes were larger among subjects reporting greater fatigue, particularly those experiencing drowsiness, local pain and dullness. These 
Table 5. Changes in immunological parameters between the beginning of day shifts and the end of night shifts grouped by age, marital status, ward assignment and hours of sleep before the night shift

\begin{tabular}{|c|c|c|c|c|c|c|}
\hline & $\begin{array}{c}\text { Number of } \\
\text { subjects }\end{array}$ & $\begin{array}{l}\text { NK cell activity } \\
\text { (E:T=20:1, \%) }\end{array}$ & $\begin{array}{c}\mathrm{CD}^{+} \\
\text {lymphocytes } \\
\text { (number/ } \mu l \text { ) }\end{array}$ & $\begin{array}{c}\mathrm{CD}^{+} \\
\text {lymphocytes } \\
\text { (number/ } \mu l \text { ) }\end{array}$ & $\begin{array}{c}\mathrm{CD}^{+} \\
\text {lymphocytes } \\
\text { (number/ } \mu l \text { ) }\end{array}$ & $\begin{array}{l}\mathrm{CD} 16^{+} \mathrm{CD} 56^{+} \\
\text {lymphocytes } \\
\text { (number } / \mu l \text { ) }\end{array}$ \\
\hline All & 57 & $-3.3(6.3)$ & $205.3(234.9)$ & $124.1(144.3)$ & $22.8(143.7)$ & $-69.7(147.8)$ \\
\hline \multicolumn{7}{|l|}{ Age (yr) } \\
\hline $23-43$ & 29 & $-4.6(6.0)$ & $290.2(213.1)^{*}$ & $173.6(125.4)^{*}$ & $68.6(130.4)^{*}$ & $-46.9(126.4)$ \\
\hline $44-59$ & 28 & $-1.9(6.5)$ & $117.4(227.1)$ & 72.7 (146.6) & $-24.5(143.7)$ & $-93.4(166.1)$ \\
\hline \multicolumn{7}{|l|}{ Marital status } \\
\hline Single & 10 & $-6.9(7.6)^{*}$ & $150.8(219.3)$ & $68.7(154.0)$ & $7.9(159.5)$ & $-74.6(165.0)$ \\
\hline Married & 47 & $-2.5(5.9)$ & $216.9(238.7)$ & $135.8(141.0)$ & $26.0(141.8)$ & $-68.7(145.8)$ \\
\hline \multicolumn{7}{|l|}{ Wards for patients with: } \\
\hline Dementia & 28 & $-5.2(6.8)^{*}$ & $220.9(247.7)$ & $142.1(141.8)$ & $15.9(167.2)$ & $-98.3(185.2)$ \\
\hline $\begin{array}{l}\text { Schizophrenia and } \\
\text { schizophreniform disorder }\end{array}$ & 29 & $-1.4(5.4)$ & $190.2(225.1)$ & $106.7(146.9)$ & 29.5 (119.5) & $-42.1(94.9)$ \\
\hline \multicolumn{7}{|c|}{ Hours of sleep before the night shift } \\
\hline $0-2.0 \mathrm{~h}$ & 34 & $-4.7(5.7)^{*}$ & $237.2(226.6)$ & $144.3(167.1)$ & $35.6(124.7)$ & $-65.7(117.8)$ \\
\hline $2.5-4.0 \mathrm{~h}$ & 23 & $-1.1(6.7)$ & $158.2(243.9)$ & $94.1(97.5)$ & $4.0(169.3)$ & $-75.7(186.3)$ \\
\hline
\end{tabular}

Values are expressed as means (standard deviation) in the category. Statistical differences were evaluated by $t$-test. *: $p<0.05$.

findings did not change after adjustment for other demographic factors or hours of sleep prior to starting the night shift. Our results demonstrate that shift work results in an accumulation of fatigue and that fatigue impacts important aspects of innate immunity, such as NK cell function. Chronic suppression of NK cell activity due to repetitive rounds of shift work could be one of the variables influencing the relationship between shift work and cance $^{20)}$. It is possible that improving the shift system to reduce fatigue could reduce the negative impact on innate immunity. Interestingly, Kobayashi et al. ${ }^{21)}$ evaluated the effects of improvements of a shift system on fatigue and immune parameters among nurses. The subjects in their report engaged in a similar pattern of shift work as the one in use at the hospital in our study. Lengthening the interval between day shifts and night shifts by introducing a half-day shift reduced fatigue; however, NK cell function was virtually identical to that seen with the former shift work pattern. Several studies have examined the relationship of fatigue and immune function, although these studies did not target shift workers. Shakhar et al. $\left.{ }^{22}\right)$ investigated the relationship between the level of fatigue and changes in immune parameters over a one-month interval among female workers engaged in day work and found that decreased fatigue and increased sleep hours resulted in an increase in NK cell activity. Studies that have investigated the impact of long work hours on immune function have identified fatigue as one of the most important factors ${ }^{23-25)}$. These studies consistently identified suppressed NK cell activity in subjects with longer work hours.
We found an increase in the number of $\mathrm{CD} 4^{+}$ lymphocytes among subjects experiencing greater fatigue. The results of several laboratory studies were not consistent for T lymphocytes. Born et al. ${ }^{11)}$ examined the diurnal rhythms of immune parameters, including lymphocyte subsets, between groups receiving either normal nocturnal sleep or total sleep deprivation. They reported that the number of T lymphocytes reached peak levels at 23:00 and nadir levels at 8:00 in both groups; however, the peak levels in the sleep-deprived group were higher than in those receiving normal nocturnal sleep. Conversely, there are also reports showing decreases in $\mathrm{CD}^{+}{ }^{+} \mathrm{T}$ cell numbers due to mental stress ${ }^{26,27)}$. Although not currently a topic of study, lymphocyte proliferation might be influenced by acute and chronic stress or by mental and physical stress. Stressors impact the normal functioning of natural and specific immunity in proportion to the magnitude and duration of the stressors ${ }^{13)}$. The fatigue levels of our subjects likely reflect both acute stress due to sleep deprivation during night shift and chronic stress due to persistent perturbations in their work/sleep schedules. The increase in $\mathrm{T}$ cell populations might be a defensive response to counter the risks of acute stress experienced during sleep deprivation. However, the increase in $\mathrm{T}$ cell populations may also have detrimental effects, since it has been suggested that some cytokines, such as IL- 6 secreted by CD4+ lymphocytes, can reactivate latent herpes virus ${ }^{28}$.

The strength of our study was that we utilized a longitudinal design to show that the effects of shift work on NK cell function and lymphocyte subsets depended on 


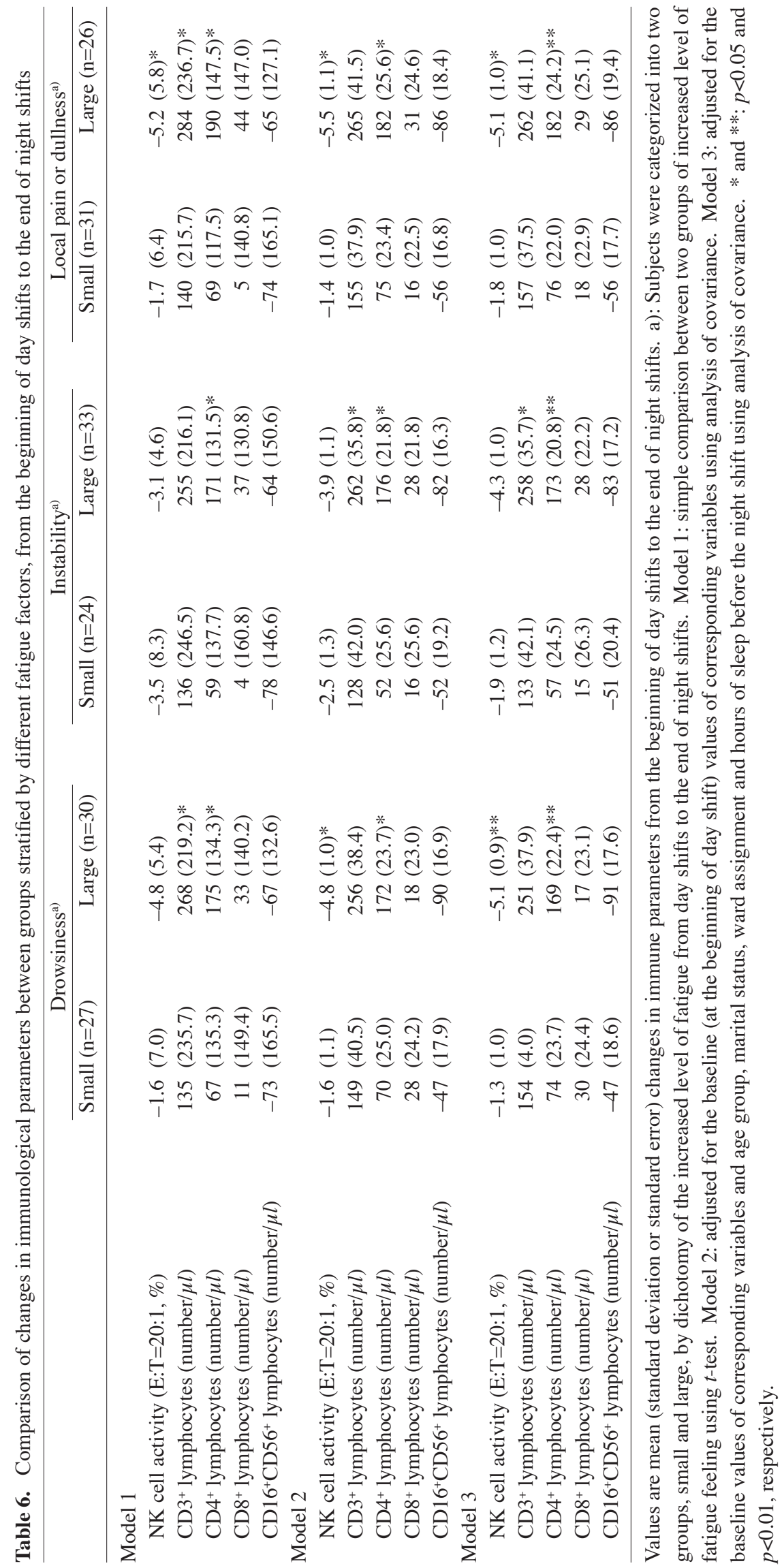


the level of fatigue caused by shift work. However, our study had some limitations. First, we studied female nurses in one hospital, although the shift system employed by this hospital is in use in other Japanese hospitals. Studies on other shift systems and other workplace settings should be conducted. Secondly, we did not evaluate the effects of chronic mental stress, although there have been many studies on the relationship between mental stress and immune function. Finally, we did not evaluate the menstrual cycles of subjects, which might influence immune function.

In conclusion, our data show that the degree of fatigue due to shift work impacts immune function. Proper management of shift systems to reduce the level of fatigue in workers is important to diminish health problems including cancer risk.

Acknowledgment: This study was supported by a grantin-aid for scientific research (14570362) from the Ministry of Education, Culture, Sports, Science and Technology of Japan. Our special thanks go to all who participated in this study.

\section{References}

1) Straif K, Baan R, Grosse Y, et al. Carcinogenicity of shift-work, painting, and fire-fighting. Lancet Oncol 2007; 8: 1065-6.

2) Costa G, Hause E, Stevens R. Shift work and cancerconsiderations on rationale, mechanisms, and epidemiology. Scand J Work Environ Health 2010; 36 : 163-79.

3) Carrillo-Vico A, Guerrero JM, Lardone PJ, Reiter RJ. A review of the multiple actions of melatonin on the immune system. Endocrine 2005; 27: 189-200.

4) Nakano Y, Miura T, Hara I, et al. The effect of shift work on cellular immune function. J Hum Ergol 1982; 11: 131-7.

5) Okamoto H, Tsunoda T, Teruya K, et al. An occupational health study of emergency physicians in Japan: health assessment by immune variables (CD4,CD8,CD56, and NK cell activity) at the beginning of Work. J Occup Health 2008; 50: 136-46.

6) Irwin M, Mcclintick J, Costlow C, Fortner M, White J, Gillin JC. Partial night sleep deprivation reduces natural killer and cellular immune responses in humans. FASEB J 1996; 10: 643-53.

7) Irwin M, Mascovich A, Gillin JC, Willoughby R, Pike J, Smith TL. Partial sleep deprivation reduces natural killer cell activity in humans. Psychosom Med 1994; 56: 493-8.

8) Matsumoto Y, Mishima K, Satoh K, et al. Total sleep deprivation induces an acute and transient increase in NK cell activity in healthy young volunteers. Sleep 2001; 24: 806-11.

9) Dinges DF, Douglas SD, Zaugg L, et al. Leukocytosis and natural killer cell function parallel neurobehavioral fatigue induced by 64 hours of sleep deprivation. J Clin Invest 1994; 93: 1930-9.
10) Heiser P, Dickhause B, Schreiber W, et al. White blood cells and cortisol after sleep deprivation and recovery sleep in humans. Eur Arch psychiatry Clin Neurosci 2000; 250: 16-23.

11) Born J, Lange T, Hansen K, Mölle M, Fehm HL. Effect of sleep and circadian rhythm on human circulating immune cells. J Immunol 1997; 158: 4454-64.

12) Friedman EM, Lawrence DA. Environmental stress mediates changes in neuroimmunological interactions. Toxicol Sci 2002; 67: 4-10.

13) Segerstrom SC, Miller GE. Psychological stress and the human immune system: a meta-analytic study of 30 years of inquiry. Psychol Bull 2004; 130: 601-30.

14) Pross HF, Baines MG, Rubin P, Shragge P, Patterson MS. Spontaneous human lymphocyte-mediated cytotoxicity against tumor target cells. IX. The quantitation of natural killer cell activity. J Clini Immunol 1981; 1: 51-63.

15) Kubo T, Tachi N, Takeyama H, et al. Characteristic patterns of fatigue feelings on four simulated consecutive night shifts by "Jikaku-sho Shirabe". Sangyou Eiseigaku Zasshi 2008; 50: 133-44(in Japanese).

16) Takeyama H, Itani $T$, Tachi N, et al. Effect of a modified ambulance night shift system on fatigue and physiological function among ambulance paramedics. J Occup Health 2009; 51: 204-9.

17) Tachi N, Itani T, Sasaki K, et al. Validity of a newly developed questionnaire for evaluating work-related fatigue feeling. Brazil: 27th International Congress on Occupational Health.

18) Kurumatani N, Koda S, Nakagiri S, et al. The effects of frequently rotating shiftwork on sleep and the family life of hospital nurses. Ergonomics 1994; 37: 9951007(in Japanese).

19) Matsumoto S, Sasaki T, Sakita M, et al. The effect of naps taken by hospital nurses during 16-hour nightshifts on their subjective fatigue feelings and subsequent sleeps. Roudoukagaku 2008; 84: 25-9 (in Japanese).

20) Imai K, Matsuyama S, Miyake S, Suga K, Nakachi K. Natural cytotoxic activity of peripheral-blood lymphocytes and cancer incidence: an 11-year follow-up study of a general population. Lancet $2000 ; 356$ : 1795-9.

21) Kobayashi F, Furui H, Akamatsu $Y$, Watanabe T, Horibe H. Changes in Psychophysiological functions during night shift in nurses influence of changing from a fullday to a half-day work shift before night duty. Int Arch Occup Environ Health 1997; 69: 83-90.

22) Shakhar K, Valdimarsdottir HB, Guevarra JS, Bovbjerg DH. Sleep, fatigue, and NK cell activity in healthy volunteers: significant relationships revealed by within subject analyses. Brain Behav Immun 2007; 21: 180 4.

23) Morikawa Y, Kitaoka-Higasiguchi K, Tanimoto C, et al. A cross-sectional study on the relationship of job stress with natural killer cell activity and natural killer cell subsets among healthy nurses. J Occup Health 2005; 47: 378-83.

24) Xu M, Miura Y, Nagao F, Muto T, Okumura K. NK cell activity and subsets of truck drivers along with related 
factors. Japanese Journal of Hygiene 1998; 53: 456-62 (in Japanese).

25) Yasuda A, Iwasaki K, Sasaki T, Oka T, Hisanaga N. Lower percentage of CD56+ cells associated with long working hours. Ind Health 2001; 39: 221-3.

26) Kawakami N, Tanigawa $T$, Araki S, et al. Effect of job strain on helper-inducer (CD4+CD29+) and suppressorinducer (CD4+CD45RA+) T cell in Japanese blue-collar

workers. Psychother Psychosom 1997; 66: 192-8.

27) Nakata A, Araki S, Tanigawa $T$, et al. Decrease of suppressor-inducer (CD4+CD45RA) T lymphocytes and increase of serum immunoglobulin $\mathrm{G}$ due to perceived job stress in Japanese nuclear electric power plant workers. J Occup Environ Med 2000; 42: 143-50.

28) Kondo K. Human herpesvirus latency and fatigue. Uirusu 2005; 55; 9-17 (in Japanese). 